Meta

Journal des traducteurs

Translators' Journal

\title{
La traduction archaïsante : Cervantes d'après M. Molho
}

\section{Robin Lefere}

Volume 39, numéro 1, mars 1994

La traduction et l'interprétation dans la Belgique multilingue

URI : https://id.erudit.org/iderudit/003454ar

DOI : https://doi.org/10.7202/003454ar

Aller au sommaire du numéro

Éditeur(s)

Les Presses de l'Université de Montréal

ISSN

0026-0452 (imprimé)

1492-1421 (numérique)

Découvrir la revue

Citer cet article

Lefere, R. (1994). La traduction archaïsante : Cervantes d'après M. Molho. Meta, 39(1), 241-249. https://doi.org/10.7202/003454ar d'utilisation que vous pouvez consulter en ligne.

https://apropos.erudit.org/fr/usagers/politique-dutilisation/ 


\title{
LA TRADUCTION ARCHAIISANTE : CERVANTES D'APRESS M. MOLHO
}

ROBIN LEFERE

Chargé de recherches au FNRS, Bruxelles, Belgique

\begin{abstract}
Maurice Molho est l'auteur de la plus récente traduction, en langue française, des deux novelas ejemplares explicitement couplées par Cervantes: El casamiento engañoso et Coloquio de los perros. Parue en édition «de poche» en 1970 (Aubier-Flammarion), elle a été récemment rééditée (1992), sur support plus riche ${ }^{1}$. Paradoxalement (pour qui ignore l'histoire de la traduction - ou faudrait-il dire sa théorie ?), cette ultime version est aussi, sur le plan de la langue, la plus archaïque de celles qui sont encore communément disponibles (principalement celles de Louis Viardot 1838, et de Jean Cassou 1949). Le lecteur découvre une traduction rapidement identifiable - sinon conceptualisable - comme «archaissante», parti qu'il acceptera sans doute volontiers, du moins jusqu'à ces points critiques où la résistance variable du texte précipite en de vraies difficultés: le «luxe» philologique (ou le souci épistémologique? Nous en discuterons) pourra alors sembler complication abusive, et même absurde dans ces quelques cas ${ }^{2}$ où le texte français n'est compréhensible qu'à la lumière du texte espagnol (donné en regard, il est vrai). Ces diverses difficultés sont en nombre suffisant pour que le lecteur revienne sur le choix de la traduction archaïsante et le remette en question.

La matière est passionnante, par la multiplicité des perspectives et problèmes qu'elle implique. Elle a pourtant été étonnamment peu étudiée ${ }^{3}$... Nous partirons de l'examen de la traduction de Maurice Molho (en particulier du corpus que constitue Le mariage trompeur) pour esquisser une réflexion systématique sur l'intérêt et les problèmes de la traduction archaïsante, en général et dans ses différentes modalités.

Si l'on cherche à préciser le statut linguistique du texte de M. Molho, on s'aperçoit rapidement que la première impression de traduction «archaïsante», globalement valable, doit être précisée et nuancée.

D'abord pour cette raison que $M$. Molho réfère sa traduction à une traduction antérieure, contemporaine de l'original espagnol:
\end{abstract}

Il a été tenu compte, dans la présente traduction, de celle du Sr d'Audiguier, qu'on a entièrement refaite, sans en altérer - du moins on l'espère - la vivacité et l'esprit, en vertu du principe que les traductions d'époque sont toujours, malgré leurs écarts à la lettre, les plus fidèles à l'âme des textes. (1970:97)

Cette note, trop courte (et trop discrète ${ }^{4}$ ), et qui curieusement disparaîtra dans l'édition de 1992, est certes peu explicite (nous en discuterons plus avant la partie «théorique»), mais aussi peu claire : une traduction refaite, même «entièrement», est davantage qu'une traduction dont on a «tenu compte» (et ne faudrait-il d'ailleurs pas tenir compte, en principe, de toutes les traductions ${ }^{5}$ ?); et que signifie, de façon précise, cet «entièrement»?

Or la clarification serait d'autant plus souhaitable qu'est engagée une question essentielle : faut-il considérer la traduction de $M$. Molho comme archaïsante, ou archaïque (refaite)?

Cependant, avant même d'effectuer une comparaison des deux traductions, il est possible de trancher: une traduction archaïque refaite, même si elle devait n'être refaite 
que ponctuellement et dans une langue parfaitement archaïque, est une traduction archaïsante, non seulement en raison du contexte de production des amendements ponctuels, mais d'abord parce que ceux-ci sont l'envers d'une réassomption générale qui exprime le choix d'un traducteur moderne. Dans cette perspective, et à la limite, la simple réédition d'une traduction archaïque suffit à convertir celle-ci en traduction archaïsante ${ }^{6}$.

L'étude linguistique du texte de $\mathbf{M}$. Molho, notamment la comparaison avec celui de d'Audiguier, montre que la traduction est largement (non «entièrement») refaite, et que son statut est donc, sans discussion possible, celui d'une traduction archaïsante. Plus précisément, cette étude explicite la nature mêlée de la traduction, puisque celle-ci est en effet constituée d'un:

- texte archaïque: directement emprunté à d'Audiguier mais modernisé minimalement (graphie, traits morphologiques), comme d'ailleurs le texte espagnol proposé en regard;

- texte archaïque modernisé (stylistiquement cette fois ${ }^{7}$ );

- texte proprement archaïsant, correspondant en vérité à différents états de langue. Avec cette singularité : à suivre Furetière, on note plusieurs hyperarchaïsmes lexicaux : mots ayant cours au XVII ${ }^{\mathrm{e}}$ siècle mais devenus rares, mots/expressions n'ayant plus cours avec la même acception et mots hors d'usage - étant particulièrement remarquables, pour ces trois catégories, les cas où $\mathrm{M}$. Molho a substitué un mot archaïque au mot courant utilisé par d'Audiguier (on pourrait ainsi parler de «surarchaïsmes» ${ }^{8}$ ).

Il ne s'agit donc pas, en dépit de l'appui sur une traduction archaïque, d'une traduction uniformément archaïsante, mais l'archaïsation n'est pas non plus de l'ordre de la superficielle patine. Le texte confond délibérément différents états de langue, proposant ainsi l'œuvre espagnole dans une langue française qui n'a, à strictement parler, jamais existé, mais qui dans l'ensemble est assez proche de la langue de d'Audiguier, et donc relativement synchronique par rapport au texte espagnol.

On arrive ainsi à la question principale : pourquoi la traduction archaissante? Quels en sont les bénéfices, ou déficits? Dans quelles circonstances et dans quelle mesure seraitelle pertinente? Nous essaierons d'articuler une réflexion d'ordre théorique qui prenne en compte différentes perspectives sur la traduction (principalement l'ontologique et la pragmatique), et examine les uns par rapport aux autres différents types de traduction archaïsante: la recherche doit s'effectuer et se nuancer en fonction d'un cadre typologique.

\section{TYPOLOGIE}

La traduction d'un texte du passé est nécessairement modernisante ou archaïsante. Est en principe archaïsante toute traduction qui ne se fait pas dans une langue strictement moderne, ce qui n'exclut pas, dans les faits, qu'une traduction soit à la fois modernisante et archaïsante (il s'agirait même du cas de traduction archaïsante le plus fréquent, $c f$. ciaprès les traductions «superficiellement» voire «librement» archaïsantes).

\section{Traduction modernisante :}

1. minimalement modernisante (modernisation exclusivement linguistique ${ }^{9}$ ):

1.1. involontairement modernisante ( $c f$. la traduction ingénue, généralement «littéraliste» ${ }^{10}$ )

1.2. résolument modernisante ( $c f$. une «réécriture»)

2. violemment modernisante ( $c f$. les «belles infidèles»).

\section{Traduction archaïsante :}

1. indirectement archaïsante (cas de la traduction archaïque refaite)

2. directement archaïsante : 
2.1. Superficiellement archaïsante (texte en fait essentiellement modernisant mais parsemé de quelques traits archaïques, le plus souvent lexicaux, rarement synchroniques par rapport au texte original)

2.2. résolument archaïsante :

2.2.1. strictement archaïsante (un seul état de langue, synchronique ou non par rapport au texte original)

2.2.2. librement archaïsante (plusieurs états de langue, avec généralement un état dominant).

\section{TRADUCTION MODERNISANTE OU ARCHAÏSANTE ? ARGUMENTS GÉNÉRAUX}

La traduction modernisante a l'avantage, du moins quand elle est écrite selon un parti «cibliste» (Ladmiral) et «transparent» (Mounin), de la parfaite familiarité linguistique, permettant ainsi au lecteur d'entrer immédiatement dans le texte, et d'oublier le «verre» de la traduction.

Cependant, cette familiarité même peut devenir gênante: certains lecteurs, les plus sensibles sans doute, seront frappés par un effet de discordance, entre la langue, leur langue, et le monde «exotique» (autre contexte spatio-temporel) avec lequel elle se trouve associée, voire dans lequel elle serait parlée ${ }^{11}$. D'où un problème de réception, et un premier argument, d'ordre pragmatique, en faveur d'une traduction archaïsante : elle évite l'effet de discordance. Davantage: elle introduit dans la réception une distance linguistique qui agit psychologiquement comme «effet de réel», favorisant ainsi l'adhésion du lecteur. À cet égard, une archaïsation superficielle, purement «tactique» ${ }^{12}$, suffirait (sauf public exceptionnel de philologues-historiens).

$\grave{A}$ supposer que la réception ne produise aucun effet de discordance, il reste que, ontologiquement, il y a discordance ou inadéquation entre une langue moderne et un monde idéologiquement et culturellement archaïques.

Mais avant même la discordance intrinsèque, perçue ou non, il y a plus évidemment celle entre l'apparence (la nature) archaïque du texte original et celle, moderne, d'une traduction non archaïsante: cette discordance, extrinsèque mais également ontologique, est immédiatement frappante (le lecteur s'attend à un stimulus archaïque).

D'où deux arguments, de type ontologique cette fois, en faveur de la traduction archaïsante : la traduction archaïsante, et de préférence indirectement ou résolument archaiisante, reproduit la nature archaïque du texte, et instaure une certaine harmonie entre état de langue et état de culture (nous préciserons), produisant ainsi un texte plus intimement cohérent (du moins sur le plan culturel).

Le troisième type d'argument, apparemment le plus simple et naïf, serait le plaisir de donner au texte une saveur proprement linguistique de passé. Argument d'ordre esthéticophilologique, mais qui se trouve avoir une double dimension pragmatique et épistémologique ( $c f$. supra).

Cette saveur, et avec elle le plein plaisir esthétique et philologique, ce n'est qu'une traduction résolument archaïsante qui peut l'apporter. Cependant, le parti même de rigueur menace de rendre la traduction difficilement compréhensible et de compromettre le bénéfice pragmatique, au point d'altérer le plaisir esthétique, voire d'irriter, et ainsi de se retourner contre lui-même. Dans le cas d'une traduction librement archaïsante, la difficulté, quoiqu'en principe moindre, serait aussi moins tolérable pour le lecteur, dans la mesure où il la sentirait plus contingente. De sorte qu'il vaudrait mieux opter soit pour une traduction strictement archaïsante (au risque, acceptable ou non selon le type d'édition et le destinataire prévus, d'une incompréhensibilité pour les non-philologues), soit pour une traduction librement archaïsante mais, qui le soit dans les limites d'une compréhensibilité par le lecteur commun. Nous verrons néanmoins que certaines difficultés pragmatiques pourraient être justifiées à partir d'une conception pragmatique de la fidélité. 
Un dernier argument, proprement philologique, serait celui de la récupération, ou de la réactualisation, du passé linguistique. Valable dans le cas d'une traduction strictement archaïsante, il l'est beaucoup moins dans celui d'une traduction librement archaïsante, créatrice d'une langue hybride; et, si l'artifice peut éventuellement réjouir le philologue ${ }^{13}$, il risque aussi de confondre et de fausser la conscience linguistique commune que l'on se proposait d'enrichir.

\section{L'ARGUMENT DE LA «FIDÉLITÉ»}

Avec la discussion antérieure d'une «discordance» ou d'une «inadéquation» ontologiques s'introduisait, implicitement, le concept essentiel de «fidélité», quoique de façon très restreinte: fidélité à la nature archaique du texte, fidélité (adéquation linguistique) à la réalité culturelle à laquelle fait référence le texte original; mais non nécessairement à l'«esprit» du texte, ni encore moins à sa «lettre», ou aux intentions qui le régiraient... La question de savoir si et dans quelle mesure la traduction archaïsante - laquelle? garantit une plus grande fidélité - à quoi? - est la plus complexe. Nous la traiterons dans la perspective essentielle d'une fidélité «au texte» (nous allons préciser), ce qui ne nous empêchera pas de considérer ultérieurement une autre perspective.

La note dont M. Molho faisait précéder, en 1970, sa traduction ( $c f$. précédemment), offre un bon point de départ, car, rapportant celle-ci à une traduction archä̈que posée en modèle, elle nous invite à examiner ce qui unit et différencie traduction archaïque et traduction archaïsante, et de discuter du statut de «modèle» de la première.

M. Molho invoque donc «ce principe que les traductions d'époque sont toujours, malgré leurs écarts à la lettre, les plus fidèles à l'âme des textes», en vertu de quoi elles constitueraient une excellente base pour une traduction (moderne) archaïsante.

Le principe semble relever du bon sens. (Notons que M. Molho n'est cependant pas très cohérent: souvent sa traduction s'écarte, sans nécessité nous semble-t-il, de celle de d'Audiguier, et parfois dans un sens archaïsant - surarchaïsant par rapport à nous ( $c f$. précédemment) -, et ainsi précieux ; laquelle préciosité risque de s'accorder d'autant moins avec l'«âme» de l'original qu'elle accroît l'effet de préciosité inhérent à toute traduction archaïsante).

Néanmoins : pourquoi une traduction d'époque serait-elle plus fidèle à l'«âme» du texte? Parce qu'un traducteur d'époque aurait nécessairement, à égalité d'aptitudes avec un moderne, une meilleure intelligence du texte? Ou pour les caractéristiques et ressources linguistiques et stylistiques du français (en l'occurrence) de l'époque? Ou pour l'interaction de ces deux raisons?

À la réflexion, le principe qu'on avait spontanément tendance à accepter se révèle, en tant que "principe», faux: on sait qu'à une même époque concourent différentes Histoires (chacune avec son histoire de la langue, de la sensibilité, des idées... - de la culture), dont la contemporanéité objective n'exclut pas les plus profondes disparités. Inversement l'on peut imaginer diverses affinités anachroniques — et ainsi, par exemple, une traduction d'Homère parfaite en ancien français ${ }^{14}$. Ce n'est donc que ponctuellement, au terme d'une comparaison de deux cultures synchroniques, de l'examen de leurs contacts (intellectuels et linguistiques notamment), qu'on pourrait conclure à une situation privilégiée pour une traduction réciproque. Mais même dans ce cas, qui serait celui de l'Espagne et de la France au début du XVII ${ }^{\mathrm{e}}$ siècle $^{15}$, il n'est pas sûr que la traduction d'époque soit mieux à même de traduire: d'excellents traducteurs d'aujourd'hui pourraient avoir des connaissances culturelles supérieures ( $\mathrm{si}$, du moins, les études historiques sont nombreuses, et qu'on en admet la validité), et de même sur le plan proprement philologique (sauf à nier les progrès de la philologie, ainsi que des théories du texte et de la traduction)... Et les premières seraient sans doute moins entachées de préjugés. 
En fin de compte, l'unique avantage certain du traducteur d'époque serait que, dans le cas privilégié où il y a affinité entre langue-source et langue-cible, et à supposer que cette affinité ait disparu avec l'évolution de la seconde (ainsi devenue «disconvenante»? cf. Molho, 1968: CLXXIV, CLXXVIII), ce traducteur disposerait d'une langue plus conforme par nature; la traduction archaïque offrirait ainsi :

- la possibilité - car ce n'est pas automatique : les contemporains devaient interpréter eux aussi - d'un rendu plus juste du «ton» (au sens imprécis de M. Molho (1968) : l'«esprit» ou l'«âme» du texte, dans leur versant linguistique);

- la possibilité - aux conditions susdites et relativement à la qualité du traducteur d'une plus grande fidélité à «la lettre», et donc, selon une conception «littéraliste» ${ }^{16}$, au Sens (littéralisme religieux), ou du moins à la «signifiance» (littéralisme littéraire).

Le premier bénéfice est discutable: la traduction de Louis Viardot, dont la langue n'est éloignée ni de celle de d'Audiguier ni de la nôtre, nous semble restituer elle aussi le ton (esprit du texte, de l'époque, qualité de voix). Et il n'est pas sûr qu'une langue moderne ne puisse restituer quelque ton que ce soit ${ }^{17}$... En fait, il n'y aurait pas impossibilité épistémologique, mais bien plutôt problème pragmatique et préjugé ontologique :

a) si l'on peut parler d'une «platitude de toutes les traductions modernes» (confinant à un «contresens généralisé»? (Molho 1968: CLXXVII)), ce n'est peut-être pas par disconvenance linguistique (et donc spirituelle) de celles-ci, mais seulement parce que, n'ayant pas la saveur linguistique du passé, elles apparaissent «lisses», sans «grain»... L'erreur serait d'incriminer une infidélité au «ton» du texte, quand il s'agit simplement d'une «infidélitée» au ton archaïque résultant d'une lecture moderne; et dans la mesure où ce ton projeté sur le texte est discordant d'avec le ton original, la langue moderne pourrait être plus fidèle à celui-ci (tout en suscitant, à d'autres égards, divers problèmes d'ordre ontologique ou pragmatique, voir précédemment).

b) La traduction moderne (y compris l'archaïsante) se trouverait, en raison de sa noncontemporanéité, à un degré d'authenticité ontologique inférieur à celui de la traduction archaïque ${ }^{18}$.

Le second bénéfice est discutable dans la mesure où le sont l'ambition littéraliste et la théorie littéraliste du sens... Problématique qu'il est évidemment impossible de traiter ici, mais par rapport à laquelle il faut prendre position, car si l'on accepte la validité du littéralisme, il s'agirait d'un argument décisif en faveur d'une traduction archaïsante (et en principe «littérale»). Très brièvement donc: la «traduction littérale» est «contradictoire dans son principe ${ }^{19}$, et en toute rigueur impossible, sinon, dans le cas de langues qui ne soient pas radicalement différentes, sous certains aspects (par exemple, la syntaxe, le rythme...). Et à supposer que l'on arrive à une relative conformité linguistique, celle-ci ne garantit aucunement une fidélité sémantique : le transfert dans un autre milieu altère la nature et la signifiance d'un stimulus apparemment conforme, permettant maints effets de sens pervers, jusqu'au contresens.

Il résulte donc que si, comme on l'a vu tout d'abord, la traduction archaïque ne dispose pas nécessairement d'un avantage interprétatif (ce pourrait même être le contraire), ses possibles atouts linguistiques ne garantissent pas non plus une fidélité plus grande à celle d'une traduction moderne. Elle ne jouit donc pas d'un statut supérieur par rapport à celleci, et, plus particulièrement, ne constitue pas pour la traduction archaïsante un modèle, mais un auxiliaire - précieux certes, mais sur lequel il serait erroné de trop s'appuyer ${ }^{20}$.

Concentrons-nous sur la traduction proprement archaïsante. Le texte superficiellement archaïsant est insignifiant sur le plan de la fidélité au texte. La traduction résolument archaïsante aurait, par rapport à la traduction archaïque, le double avantage de pouvoir 
choisir l'état de langue le plus adéquat, et de disposer de moyens philologiques plus élaborés. Ces avantages ne lui évitent pas de tomber sous le coup d'une critique du littéralisme, et d'ainsi voir sapé l'argument qui à lui seul aurait suffi à la justifier: celui de plus grande fidélité (éventuelle et conditionnelle) à la lettre. De plus, ces avantages sont négligeables en regard de l'important déficit ontologique...

En effet, la traduction librement archaïsante, comme celle de M. Molho, mêle différents états de langue. Or le recours à différents états de langue implique que l'on écrive dans un rapport d'extériorité qui compromet la constitution d'une parole, et ainsi d'un texte littéraire ${ }^{21}$. Ensuite, le texte produit est linguistiquement disparate, asystématique, et n'accède donc pas véritablement au statut de texte.

La traduction strictement archaïsante serait ontologiquement supérieure dans la mesure où son homogénéité est conforme à la nature de la langue et du texte. Mais cette force est aussi sa faiblesse: car si l'unité de langue permettrait en principe d'écrire à l'intérieur de cette dernière, et d'élaborer une véritable réénonciation, la langue visée est aussi, comme totalité systématíque, radicalement coupée de l'actualité (beaucoup plus nettement qu'une traduction librement archaisante), et inaccessible comme langue vivante (même pour un philologue exceptionnel). La traduction strictement archaïsante produira donc, au mieux, un simulacre de parole - en réalité : une restitution archéologique ${ }^{22}$.

Comme annoncé au début de cette section consacrée à la «fidélité», il nous reste à réenvisager celle-ci selon une perspective non adoptée jusqu'ici : la fidélité à la réception.

Non certes la réception du lecteur espagnol ou français de l'époque - réception difficilement connaissable, même dans ses grands traits, et en tout cas impossible à ressusciter (il faudrait une stratégie violemment modernisante, une actualisation spéculative qui sacrifierait à sa quête d'une fidélité chimérique toutes les autres fidélités) -, mais celle du lecteur espagnol contemporain, et exclusivement sous un aspect: la distance linguistique au texte. Ce serait selon nous le seul cas où le principe de fidélité à l'effet, érigé en principe général et non limité à résoudre des problèmes ponctuels, soit acceptable (mais non sans aléas: suppose l'identité du texte lu par le «lecteur-source» contemporain et du texte traduit; périssabilité extrême si le rythme d'évolution des langue source et langue cible est différent). Ainsi entendue, la fidélité à la réception ne constitue qu'un argument secondaire en faveur d'une traduction archaissante et doit, de toute façon, être subordonnée à la fidélité au texte en lui-même. Cependant, le principe pourrait contribuer à justifier le choix de la traduction résolument archaïsante, ainsi que des choix ponctuels (y compris des "complications»). Et ce, en contrôlant l'archaïsation par deux règles :

- à un moment $X$, et relativement aux lecteurs respectifs, le degré d'archaïsme de la traduction doit être fonction du degré d'archaïsme, à ce moment, du texte original ;

- la traduction doit s'essayer à reproduire le niveau et le type de difficultés que rencontre le lecteur-source d'aujourd'hui.

\section{CONCLUSION}

Soit un texte du passé à traduire, et l'alternative première entre traduction modernisante ou archaïsante, rien ne permet de trancher catégoriquement en faveur de l'un ou l'autre type.

La traduction minimalement modernisante est plus aisément et communément lisible, et ainsi plus fonctionnelle; elle est aussi, du point de vue du ton, plus naturelle. Cependant, son parti d'ignorer le problème temporel ne supprime pas celui-ci: elle présente une discordance ontologique externe et interne qui compromet le statut du texte, et risque de produire un «effet de discordance», ruinant le bénéfice pragmatique. 
L'option archaïsante se démultiplie en divers types archaïsants, dont nous avons essayé d'explorer et de contraster les possibilités respectives. La traduction superficiellement archaïsante est sans doute la plus fréquente. Sa prudence ou sa paresse font qu'elle est la moins riche, mais aussi la moins discutable: elle permet un bénéfice pragmatique (un positif «effet de réel» se substituant à l'«effet de discordance»), sans presque de contrepartie (l'hétérogénéité textuelle est négligeable, précisément dans la mesure où le sont les bénéfices ontologique et philologique). La traduction résolument archaïsante est, sur le plan théorique, la plus engagée et la plus problématique; et donc la plus riche, sans être pour autant la plus fondée. Tentant de surmonter l'éloignement temporel (l'inadéquation linguistique et le déficit ontologique dont celui-ci serait responsable), elle tend vers le modèle, existant ou hypothétique, d'une traduction archaïque. Ce faisant, et contrairement à ce que l'on pourrait penser ( $c f$. notamment $\mathrm{M}$. Molho), son bénéfice majeur ne réside pas dans une possibilité de fidélité plus grande au texte — en réalité, comme pour la traduction archaïque, une possibilité conditionnelle et discutable de plus grande fidélité au «ton» ou à la lettre -, mais dans le double bénéfice philologique et ontologique (respect de l'apparence archaïque du texte, production d'une certaine adéquation) qu'elles ajoutent au bénéfice pragmatique. Ces bénéfices ont leur prix, se révèlent dès lors ambivalents, au point de compromettre l'option: la traduction librement archaïsante propose une traduction qui peut être philologiquement aussi absurde et fallacieuse que savoureuse et instructive, et dont l'hétérogénéité compromet le statut ontologique du texte; quant au parti de traduction strictement archaïsante, ce serait son homogénéité même de langue morte qui pose problème (philologique, ontologique et pragmatique)...

Si la discussion théorique apparaît donc davantage indicative que prescriptive, elle impose du moins que chacun choisisse en connaissance de cause, et permet de critiquer telles options : pour leur incompatibilité avec le but qu'elles annoncent, pour leur nonpertinence par rapport au type de texte ou au contexte de publication.

En ce qui concerne la traduction de $\mathrm{M}$. Molho, si on peut lui reprocher de n'être accompagnée d'aucune ou presque aucune (selon qu'il s'agit des éditions de 1992 ou 1970) introduction explicative (que réclameraient et l'option et le type d'édition); si l'on peut discuter - comme toujours - de nombreux choix ponctuels; elle présente globalement tous les avantages et inconvénients de la traduction librement archaïsante, mais avec sans doute une prédominance des premiers. Ceci en raison de diverses circonstances qui garantissent la pertinence du choix. En effet, l'appui sur la traduction archaïque réduit l'hétérogénéité linguistique et ses effets négatifs (sur les plans philologique et ontologique), en même temps qu'il permet une certaine qualité de parole. Ensuite, la déperdition de signifiance, corrélative de la déperdition structurelle inhérente à toute traduction mais particulièrement à la traduction librement archaïsante (quoiqu'ici de façon réduite), nous semble tolérable si l'on admet que le «Casamiento engañoso» est un texte essentiellement narratif, qui ne souffre donc pas trop des altérations de sa structure sémantique. En ce qui concerne le problème le plus manifeste, celui de la difficulté linguistique, il faut reconnaître une certaine complaisance dans le parti archaïsant ( $c f$. les surarchaïsmes), qui multiplierait plutôt les obstacles. Cette complication linguistique est cependant acceptable dans la mesure où elle est assumable par le destinataire visé par l'édition (partielle, d'à peine deux «nouvelles exemplaires», bilingue, incluant un long essai de critique littéraire): philologues et étudiants. Enfin, le parti même d'une telle édition - disons «ésotérique» - se trouve totalement justifié par le fait qu'il existe des traductions plus accessibles, et de qualité (Viardot, par exemple). 


\section{Notes}

1. L'édition de 1992 est inférieure à celle de 1970 : les coquilles sont nombreuses, et l'introduction, nouvelle mais plus brève ( 15 pages au lieu de 80 ), ne comporte aucune indication sur la traduction.

2. Comparer avec le texte espagnol (la pagination est celle de l'édition de 1992, désormais la seule disponible) : «toute appareillee» (30), «en fera la raison à» (31), «s'aller colporter au bec des moyenneurs de mariage» (37), «galant à toute reste» (51)...

3. Nous n'avons trouvé aucune monographie. Il y a cependant des pages remarquables chez Émile Littré (1863:312-317 et 407-420) et surtout Georges Steiner (1976:334-353), ainsi que quelques critiques très judicieuses de la part de Henri Meschonnic (1986). L'histoire de la traduction archaïsante est brossée dans Susan Bassnett-Mc Guire (1980), évoquée chez Georges Mounin (1955).

4. Elle constitue le dernier paragraphe d'une page intitulée «Bibliographie sommaire»... faisant suite à 80 pages de «Remarques sur le Mariage trompeur et Colloque des chiens»! Semblablement, les comptes rendus de l'édition de M. Molho (Arnaud 1970, Avalle-Arce 1973) se concentrèrent sur l'introduction et ne firent, sur la traduction même, que quelques appréciations très générales ou critiques de détail.

5. Il est en particulier étonnant que ne soit pas cité Jean Cassou, qui écrivait à propos de sa traduction : $\ll(. .$. recouru à toutes les ressources que la langue française nous offre depuis le XVI ${ }^{e}$ siècle; j'étais même remonté un peu plus haut afin de trouver des équivalents aux termes d'argot picaresque de Cervantes.» (1949: 1066). On trouve chez J. Cassou maints choix qui anticipent ceux de M. Molho, y compris quelques «trouvailles» (comme «les surarchaîsmes»: «cuidait engeigner», «papegeais», $1992: 47$ et 55).

6. Que la nature intrinsèque puisse radicalement changer en fonction de la modification d'extrinsèques circonstances - davantage : en fonction de la connaissance contingente d'une modification —, c'est scandaleusement paradoxal... On sait que le relativisme de Borges a magistralement exploité le paradoxe, dès 1937 («Pierre Ménard, autor del Quijote»).

7. Modernisation stylistique dès le titre («Le mariage trompeur» et non plus «Le trompeur mariage»), à laquelle s'en ajoute une d'ordre esthétique: suppression du sous-titre ou épigraphe insérés par d'Audiguier («Description d'un mariage Trompeur, où les ruses des mauvaises femmes sont contenues»).

8. Surarchaïsmes : «a clair» (29), «tant seulement» (31), «la judiciaire» (37), «la fourbe» (43), «feintise» (43), «papegeais» $(55) \ldots$

9. À noter que si la modernisation exclusivement graphique et morphologique ne constitue pas une traduction, le cas devient limite quand l'éditeur prétend moderniser aussi la ponctuation: il touche alors au rythme du texte, c'est-à-dire à son essence même.

10. Nous serons amené à critiquer l'ambition de littéralité, mais ce qui nous importe ici, c'est faire remarquer qu'une traduction «littéraliste» d'un texte ancien peut produire, involontairement, un «effet archaïque», que l'archaïsme (d'ordre syntaxique) soit réel ou seulement dû à une interprétation par le lecteur de l'étrangeté du texte. Se constituerait ainsi un cas-limite entre traduction modernisante et traduction archaïsante; et si l'archäisme est réel, la traduction en principe modernisante se convertit inopinément en traduction archaïsante.

11. L'argument vaudrait aussi contre la traduction intra-linguistique : dans ce cas, il faudrait soit ne pas moderniser, soit moderniser vers un état antérieur de la langue. Par ailleurs, noter que la discordance temporelle serait beaucoup plus sensible que la discordance spatiale (laquelle, par ailleurs, affecte presque toute traduction).

12. G. Steiner imagine un autre «tactical motive»:

By archaicising his style he [l'auteur] produces a déjà-vu [...] exchanging an obtrusive geographocallinguistic distance for a much subtler, internalized distance in time. (1976: 347)

13. Commentant Rudolf Borchardt, G. Steiner écrit :

By virtue of "creative retransformation" (Rückverwandlung), the translator could propose, indeed enact an alternative development for his own language and culture $[. .] ..(1976: 341)$

14. Voir É. Littré (1863 : 312-313), citant E. Egger ; et, prospectivement, G. Steiner :

[...] there are texts wich we cannot yet translate but which may, through linguistic changes, through a refinement of interpretative means, through shifts in receptive sensibility, become translatable in the future. (1976: 249; voir aussi Meschonnic, $1972: 51$ )

15. Cf. M. Molho évoquant son plaisir à lire la traduction du Quijote par César Oudin :

Ce plaisir a sa source dans une certaine conformité des habitudes espagnoles et françaises d'écriture et de pensée en un même moment des deux civilisations. (1968: CLXXIII).

Extrait du chapitre «Des présentes traductions», que M. Molho écrivit pour son introduction aux Romans picaresques espagnols. C'est ici que l'on pourrait trouver un cadre explicatif à la note de 1970.

16. Nous définirions le littéralisme: une théorie du sens qui conçoit celui-ci comme inhérent à la lettre (à la «forme») de telle manière que toute opération de traduction, voire de simple paraphrase, ferait perdre l'essentiel du sens (et non pas seulement des nuances et «richesses» qui seraient de l'ordre du «supplément de sens»). Théorie qui s'enracine dans une conception sacralisante de la lettre, qui, sur le plan de la traduction, implique soit la récusation de celle-ci, soit une pratique de traduction la plus «littérale» possible. 
17. Le ton, au sens fort (intime, existentiel), pourrait même n'être traduisible que dans la langue moderne, car celle-ci seulle permet une véritable réénonciation ( $c f . \mathrm{H}$. Meschonnic et les notes 21 et 22).

18. La traduction moderne d'un texte ancien conduirait au dernier stade du processus de dégradation ontologique qu'impliquerait la traduction : du texte original à la copie ( $c f$. Berman 1986:72), détoumé de son espace et de son public naturels, arraché enfin à son époque. D'où le désir de remonter le cours, notamment par une traduction archaïsante, ou dans la volonté de fidélité à la réception du texte original (cf. plus avant), fût-elle contemporaine.

19. [...] car les langues étant différentes (à tous les niveaux), il est contradictoire de vouloir les traduire tout en les conservant. (Hurtado Albir, 1992 : 222-223)

20. M. Molho ne commet normalement pas cette erreur (en dépit du «principe» qu'il invoquait), mais parfois maintient des mots de d'Audiguier qui, en raison de leur évolution sémantique, sont très trompeurs : «succès» (31), «gaillardise» (33) ...

21. Cf. H. Meschonnic :

[...] même des réussites ponctuelles n'empêchent pas le traduire de passer d'une poétique à une stylistique : il n'y a plus de système du texte, il n'y a plus qu'un agglomérat composite. On a perdu la cohérence interne d'une énonciation. (1986:78)

22. L'archaïsme [...], quand il fait tout le système de traduction, remplace le rapport à un sujet de réénonciation (qui est dans le discours) par un énoncé de langue sans sujet. (Meschonnic, 1986: 78)

\section{RÉFÉRENCES}

BASSNETT-Mc GUIRE, Susan (1980) : Translation Studies, London \& New-York, Methuen.

BERMAN, Antoine (1986): «L'essence platonicienne de la traduction», Revue d'Esthétique, 12, pp. 63-73.

BORGES, Jorge Luis (1989) : Obras completas, I, Barcelona Emecé.

CERVANTES, Miguel de (1640): Les Nouvelles, Paris, Jeremie Bouillerot (trad. par F. de Rosset pour les six premières et par le $\mathrm{Sr}$ d'Audiguier pour les suivantes).

CERVANTES, Miguel de (1838): Les Nouvelles exemplaires, Paris, J. J. Dubochet et Cie (trad. de Louis Viardot).

CERVANTES, Miguel de (1949): Don Quichotte. Nouvelles exemplaires, Paris, Gallimard (édition établie, revue et annotée par Jean Cassou).

CERVANTES, Miguel de (1970): Le Mariage trompeur et Colloque des chiens, Paris, Aubier-Flammarion (Édition bilingue. Présentation et traduction de Maurice Molho), Rédité en 1992, («Domaine hispanique bilingue»).

Comptes rendus : Émile Arnaud, dans Cahiers du monde hispanique et luso-brésilien (Caravelle), 1970; J. B. Avalle-Arce, dans Hispanic Review, 1973.

FURETIËRE, Antoine (1690) : Dictionnaire universel, Rotterdam.

HURTADO ALBIR, Amparo (1990): La notion de fidélité en traduction, Paris, Didier.

LITTRÉ, Émile (1863) : Histoire de la langue française, I, Paris, Didier.

MESCHONNIC, Henri (1972): «Propositions pour une poétique de la traduction», Langages, 28.

MESCHONNIC, Henri (1986) : «Alors la traduction chantera», Revue d'Esthétique, pp. 75-80.

MOLHO, Maurice (1968): «Des présentes traductions», Romans picaresques espagnols, Paris, Gallimard, pp. CLXXII-CLVIII.

MOUNIN, Georges (1955): Les belles infidèles, Paris, Gallimard.

STEINER, Georges (1976) : After Babel, Oxford University Press. 